\title{
„Hochwasserrisikotool“ - GIS-basierte Methodik zur Bestimmung von Hochwasserrisiken nach den Vorgaben der EU-Hochwasserrichtlinie
}

Der Umgang mit Naturgefahren hat in Österreich in Bezug auf die Gefahrenabwehr eine lange Tradition. Der rasante Ausbau von Siedlungen, Verkehrswegen und Infrastruktureinrichtungen erfordert eine Ergänzung der bestehenden Konzepte zum Schutz vor Naturgefahren um ein risikoorientiertes Naturgefahrenmanagement. Seit 2007 fordert zudem die EU-Hochwasserrichtlinie (EUHWRL) eine Auseinandersetzung mit den naturgefahreninduzierten Risiken. Zweck der Richtlinie ist es, einen Rahmen für die Bewertung und das Management von Hochwasserrisiken zur Verringerung der hochwasserbedingten nachteiligen Folgen auf die menschliche Gesundheit, die Umwelt, das Kulturerbe und wirtschaftliche Tätigkeiten in der Gemeinschaft zu bilden (Artikel 1, Richtlinie 2007/60/EG).

Um die Ziele der EU-HWRL zu erreichen und damit ein integrales Hochwasserrisikomanagement zu etablieren, haben die Mitgliedstaaten folgende Bewertungen und Ausweisungen durchzuführen (Abb. 1).

Der Optimierung und Ergänzung der bestehenden Konzepte zum Schutz vor Naturgefahren widmet sich im Projekt „Naturgefahren Kärnten“ eine interdisziplinäre Expertenarbeitsgruppe aus den Abteilungen für Raumplanung, Forstwesen, Schutzwasserwirtschaft, Geologie und Bodenschutz des Amtes der Kärntner Landesregierung, des Forsttechnischen Dienstes der Wildbach- und Lawinenverbauung, Sektion Kärnten sowie des Studienbereichs Geoinformation der Fachhochschule Kärnten. In einer ersten Projektphase (2004-2006) wurde eine flächendeckende Geodateninfrastruktur für Naturgefahren und eine internetbasierte räumliche Darstellung der Naturgefahren in Form einer digitalen Gefahrenhinweiskarte erarbeitet. Damit konnte eine signifikante Effizienzsteigerung bei der behördenübergreifenden Zusammenarbeit und Beschleunigung bei Verwaltungsabläufen,

Amt der Kärntner Landesregierung, Abteilung 18, Klagenfurt

2 Abenis Alpinexpert GmbH, Bozen (I)

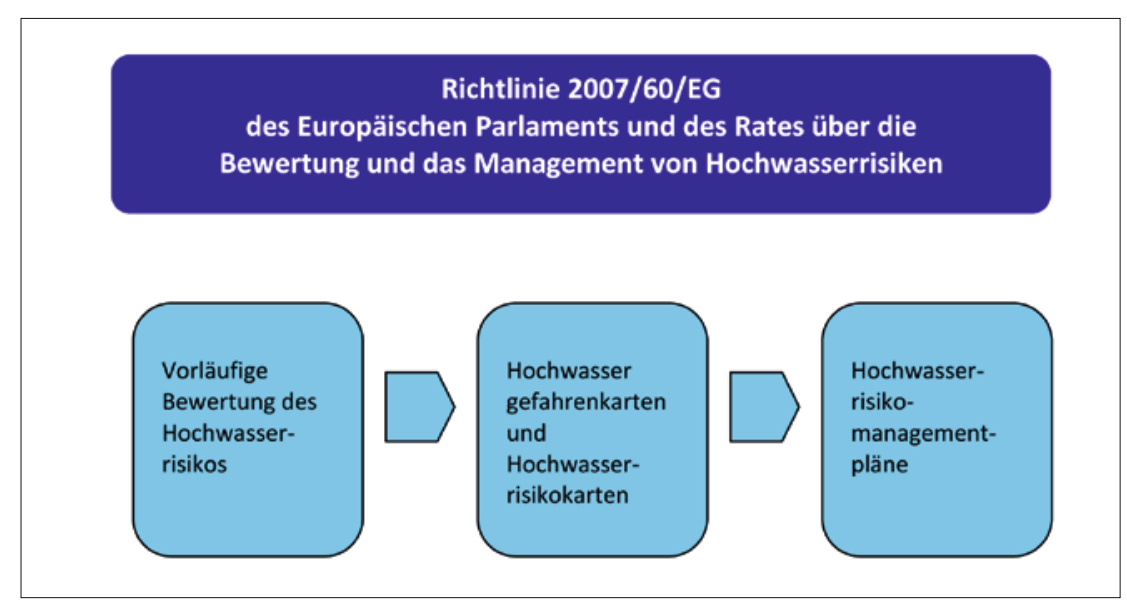

Abb. 1: Instrument der EU-Hochwasserrichtlinie (nach Pleschko et. al., 2009).

Experten-Gutachten und fachlichen Auskünften erzielt werden. Darüber hinaus dienen diese Daten der Information der Öffentlichkeit über potenzielle Gefahrengebiete (Seymann et al. 2009).

Die zweite Phase des Projektes „Naturgefahren Kärnten“ setzt den erfolgreich eingeschlagenen Weg mit den Schwerpunkten sektorale und interdisziplinäre Gefahren- und Risikoanalyse fort und leistet Vorarbeiten für die Umsetzung der EUHWRL.

Die EU-HWRL machte eine Abstimmung der nationalen Methoden und Planungsinstrumente mit jener auf EU-Ebene notwendig. Dabei wurden die bestehenden Datengrundlagen zur Bewertung der Hochwassergefahr wie der Gefahrenzonenplan und die Hochwasser-Anschlaglinien als Grundlage für die vorläufige Bewertung des Hochwasserrisikos und für die Erarbeitung der Hochwasser-Gefahrenkarten und Hochwasser-Risikokarten aufbereitet. Eine zentrale Rolle spielt in diesem Zusammenhang das Instrument der Gefahrenzonenplanung. Ein moderner Gefahrenzonenplan enthält alle wichtigen Daten (Anschlaglinien gewisser Jährlichkeiten, Bewertung der Funktion der Rückhalteräume, freizuhaltende Rückhalte- und Maßnahmenflächen) und es können für jene Gebiete nach Artikel 4 und 5 der EU-HWRL Hochwassergefahren, Risikokarten und Risikomanagementpläne daraus entnommen bzw. abgeleitet werden. Der „neue Ansatz“ im Rahmen der EU-HWRL ist jener der flächendeckenden Analyse des Risikos, wobei Geoinformationsdaten dafür grundsätzlich am besten geeignet sind.

Aufgrund der Vielzahl und der unterschiedlichen Struktur der zur Verfügung stehenden Datengrundlagen ist die Durchführung einer GIS-basierten Expositionsanalyse mit einem erheblichen Zeitaufwand verbunden. Die Strukturierung der Vorgehensweise und die Implementierung in einer automatisierten Prozedur erlaubt die Einsparung von zeitlichen Ressourcen. Das Ziel des im Nachfolgenden beschriebenen Verfahrens war es, eine Prozedur für die Analyse der Hochwassergefahr ausgesetzten Siedlungsbereiche und Infrastrukturanlagen auf Basis einer bestehenden Datengrundlage zu erarbeiten. Die Prozedur soll für einen abgegrenzten Untersuchungsraum automatisiert einen GIS-Datensatz für die Bewertung des Schadenpotenzials erstellen und eine Analyse der potenziellen Schäden ermöglichen. Der Auftrag wurde im Rahmen des WP 5 Hazard Mapping des Interreg-Alpine-Space-Projektes „AdaptAlp - Adaptation to Climate Change in the Alpine Space" durchgeführt.

\section{Methodik}

Um die Schadenpotenzial- und Risikoanalyse einheitlich und zeitsparend in vielen 


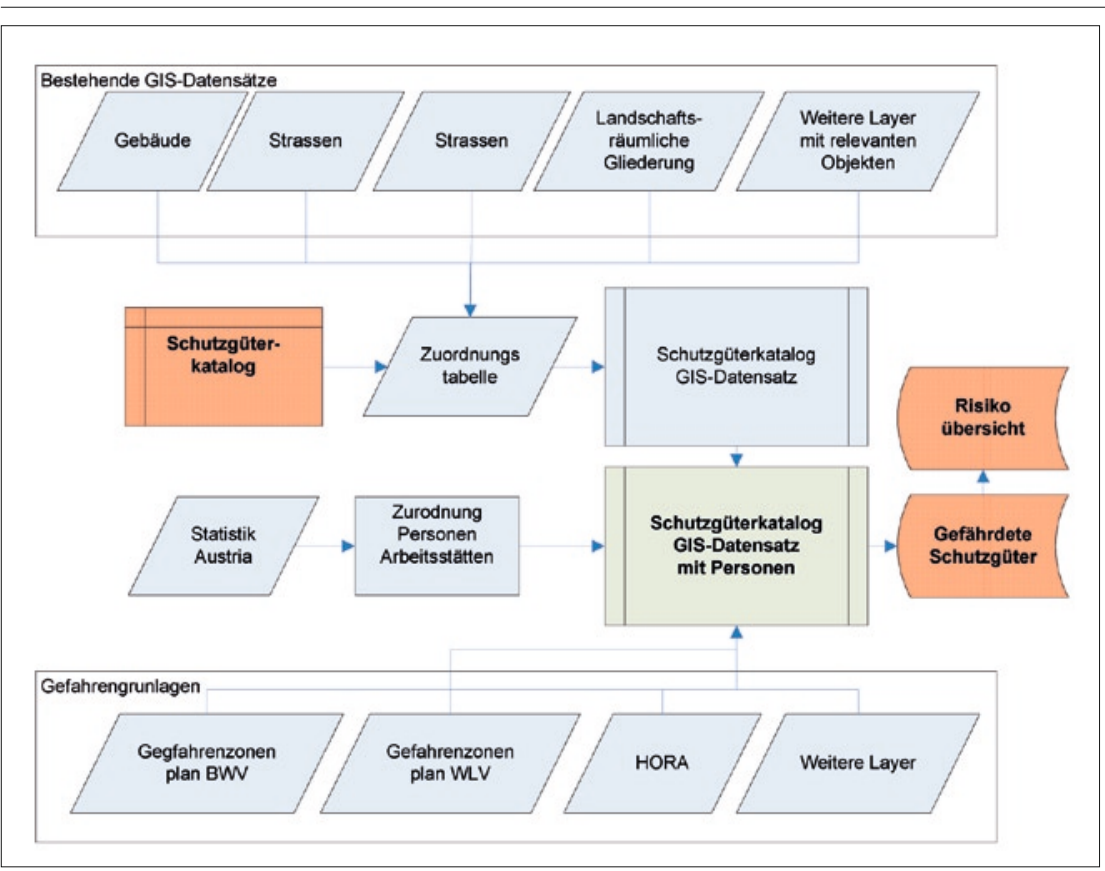

Abb. 2: Schema der Erstellung des GIS-Datensatzes der gefährdeten und schützenswerten Objekte auf Basis des Schutzgüterkataloges und der bestehenden räumlichen Datengrundlagen und die Überlagerung mit den Gefahrenkarten.

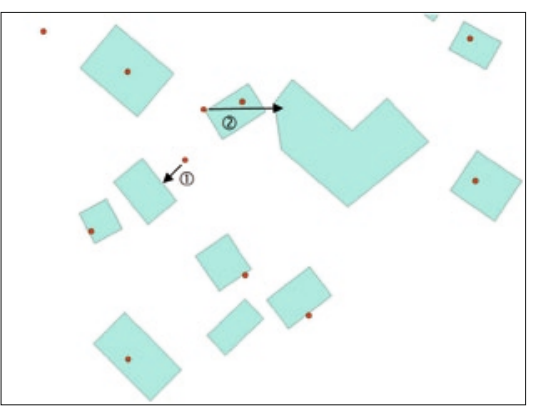

Abb. 3: Zuordnung Gebäude- und Wohnungsregister (GWR-II-Punkte) und Gebäude. Die Pfeile symbolisieren die Zuordnung der Punkte zu den Gebäuden.

Gebieten durchführen zu können, wurde ein Verfahren entwickelt, das diese Arbeit automatisiert erledigt. Das Verfahren wurde in eine GIS-Applikation eingebaut, die als Extension in die verwendete Desktop-GIS-Software der Bundeswasserbauverwaltung und der Wildbach- und Lawinenverbauung geladen werden kann. Die GIS-Applikation besteht aus drei Prozeduren. Die erste Prozedur erarbeitet den räumlichen Datensatz des Schutzgüterkataloges für ein abgegrenztes Untersuchungsgebiet auf Basis der existierenden Grundlagendaten. Die zweite Prozedur überlagert den räumlichen Schutzgüterkatalog mit den bestehenden Datensätzen der verschiedenen Gefahrenkarten und Gefahrenzonen im Sinne einer Expositionsanalyse. Dabei werden die den einzelnen Gefahrenarten ausgesetzten Objekte ermittelt. Die dritte Prozedur berechnet tensatzes des Schutzgüterkataloges ist die Attributierung des Datensatzes der Gebäudegrundrisse mit Informationen aus verschiedenen Datensätzen wie des $\mathrm{Ge}$ bäude- und Wohnungsregisters, des Datensatzes der Schulen oder anderer wichtiger Objekte, die zumeist als Punktdaten vorliegen. Alle Punktdatensätze werden über die räumliche Lage mit den Gebäudegrundrissen verknüpft. Das heißt, wenn ein Punkt des Adressdatensatzes auf ein Gebäude des Gebäudelayers fällt, wird er diesem zugeordnet und die im Adressdatensatz (Punkt) enthaltene Information wird über die Zuordnungstabelle umklassifiziert und dem Gebäudedatensatz (Fläche) zugewiesen. Vielfach fallen die Punkte jedoch nicht direkt auf das betreffende Gebäude, sondern etwas daneben. In diesem Fall wird jeder Adresspunkt innerhalb einer maximalen Suchdistanz von $20 \mathrm{~m}$ dem nächsten Gebäude zugeordnet (Abb. 3). Diese Suchdistanz wurde aufgrund von statistischen Analysen festgelegt, mit diesem Wert können $95 \%$ aller Gebäudegrundrisse durch den Adressdatensatz klassifiziert werden. In einigen Fällen kommt es vor, dass einem Gebäude mehrere Punkte zugeordnet werden. Dies ist dann der Fall, wenn zusammengebaute Häuser als ein großes Gebäude kartiert wurden. Um diese Spezialfälle zu berücksichtigen, wird die Fläche des jeweiligen Gebäudes für jede Nutzungsart einmal kopiert, um die Überlagerung des Gebäudes für jeden Nutzungstyp mit der Gefahreninformation zu erlauben. Bei der Analyse wird die Fläche des Gebäudegrundrisses durch die Anzahl der zugeordneten Punkte geteilt und so jeder Gebäudekategorie nur ein entsprechender Teil der Grundrissfläche zugeordnet.

Die Anzahl der betroffenen Personen und Arbeitsplätze kann mithilfe des Statistik-Austria-Pakets A ermittelt werden. Dieses gibt in einem 125-m-Raster Auskunft über die Anzahl der Haupt- und Nebenwohnsitze sowie über Anzahl der Arbeitsstätten und Beschäftigten. Für jede Rasterzelle des Statistik-Datensatzes werden alle Gebäude mit Wohnfunktion ausgewählt, die in dieser Rasterzelle liegen. Die Anzahl der Einwohner dieser Rasterzelle wird anschließend durch die Anzahl der Adresspunkte mit Wohnfunktion dividiert und dieser Wert anschließend dem Adresspunkt zugewiesen. Das Vorgehen wird für die Gebäude mit gewerblicher Funktion wiederholt.

Bei der Zuordnung der Attribute der Adressdaten (Punkte) an die Gebäudegrundrisse (Polygone) können nach dieser 


\begin{tabular}{|c|c|c|c|c|}
\hline GIS Datensatz & $\begin{array}{l}\text { Menschliche } \\
\text { Gesundheit }\end{array}$ & Umwelt & Kulturerbe & $\begin{array}{l}\text { Wirtschaft- } \\
\text { liche Tätigkeit }\end{array}$ \\
\hline Gebäudegrundrisse & $x$ & & $\times$ & $x$ \\
\hline Gebäude- und Wohnungsregister & $x$ & & $\times$ & $\times$ \\
\hline Schulen & $x$ & & & \\
\hline Landschaftsräumliche Gliederung & & & & $x$ \\
\hline Statistik-Austria-Paket A & $x$ & & & $x$ \\
\hline Navteq 2007 Points of Interest & $x$ & & $x$ & $x$ \\
\hline Nationalpark & & $x$ & & \\
\hline Naturschutzgebiete & & $x$ & & \\
\hline Schutz- und Schongebiete & & $x$ & & \\
\hline Ramsar-Gebiete & & $x$ & & \\
\hline Vogelschutz- und FFH-Gebiete & & $x$ & & \\
\hline Straßennetz KAGIS Land Kärnten & $x$ & & & $x$ \\
\hline Straßen- und Bahnnetz Navteq & $x$ & & & $x$ \\
\hline $\begin{array}{l}\text { Stromleitungen ( } 20 \mathrm{KV} \text { und } \\
110 \mathrm{KV} \text { ) - KAGIS }\end{array}$ & $x$ & $x$ & & $x$ \\
\hline $\begin{array}{l}\text { Wasserinfrastrukturanalagen - } \\
\text { WIS - KAGIS }\end{array}$ & $x$ & $x$ & & $x$ \\
\hline Gasleitungen KAGIS & $x$ & $x$ & & $x$ \\
\hline
\end{tabular}

Prozedur auch die Personen, Arbeitsstätten und Beschäftigten den Gebäuden zugeordnet werden (vgl. oben). Die Zuweisung der Information aus dem Statistik-Datensatz zum Datensatz der Adressdaten erfolgt innerhalb der Prozedur, in den Ausgabedatensatz geschrieben werden nur die auf das Gebäude aggregierten Daten. Diese bilden Mittelwerte über das gesamte Gebiet der Rasterzellen des Statistik-Datensatzes. Die Datenschutzvorgaben des Statistik-Datensatzes werden damit nicht tangiert. Dieses Vorgehen erlaubt im Vergleich zu einer Annahme von Durchschnittswerten je Gebäudetyp eine detailliertere Quantifizierung von Personen in Gebäuden.

Nachdem die Gebäudegrundrisse mit Information über den Gebäudetyp und die Anzahl der Personen und Beschäftigten erweitert wurden, werden die weiteren flächenhaften Datensätze in die Ausgabendatenbank geschrieben. Um die Gebäude herum wird als erstes die Siedlungsfläche und alle damit verbundenen Kategorien dem GIS-Datensatz des Schutzgüterkatalogs hinzugefügt. Diesem

\section{Gemeindesteckbrief}

Gemeinde Dellach im Drautal
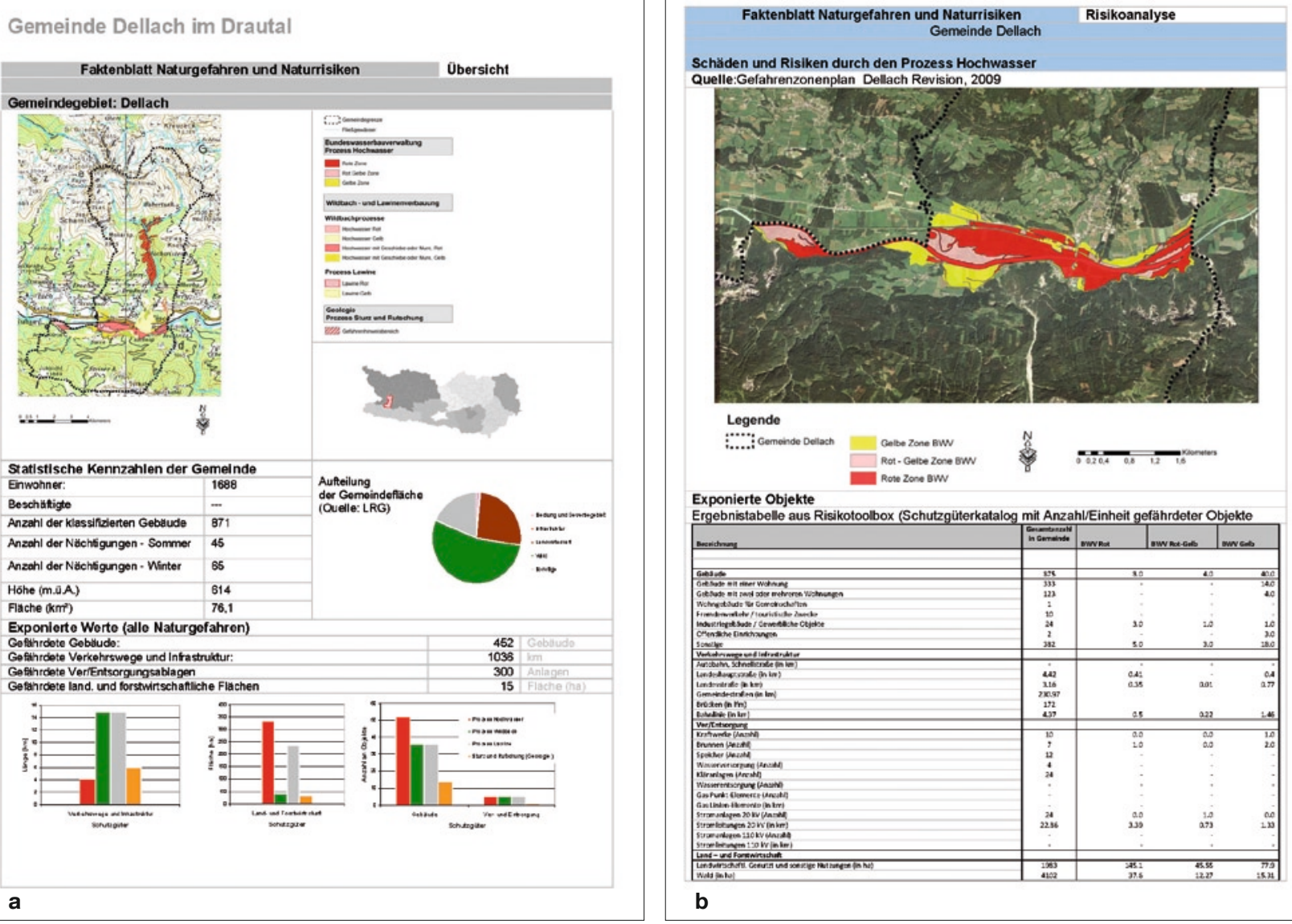

b

Abb. 4: Gemeindesteckbrief der Gemeinde Dellach im Drautal (a) allgemeine statistische Daten und Überblick über die potenziellen Schäden durch die Prozesse Hochwasser, Wildbach, Lawine, Rutschung und stürzende Ereignisse (Geologie) und (b) Detailangaben - Schäden und Risiken durch den Prozess Hochwasser. 


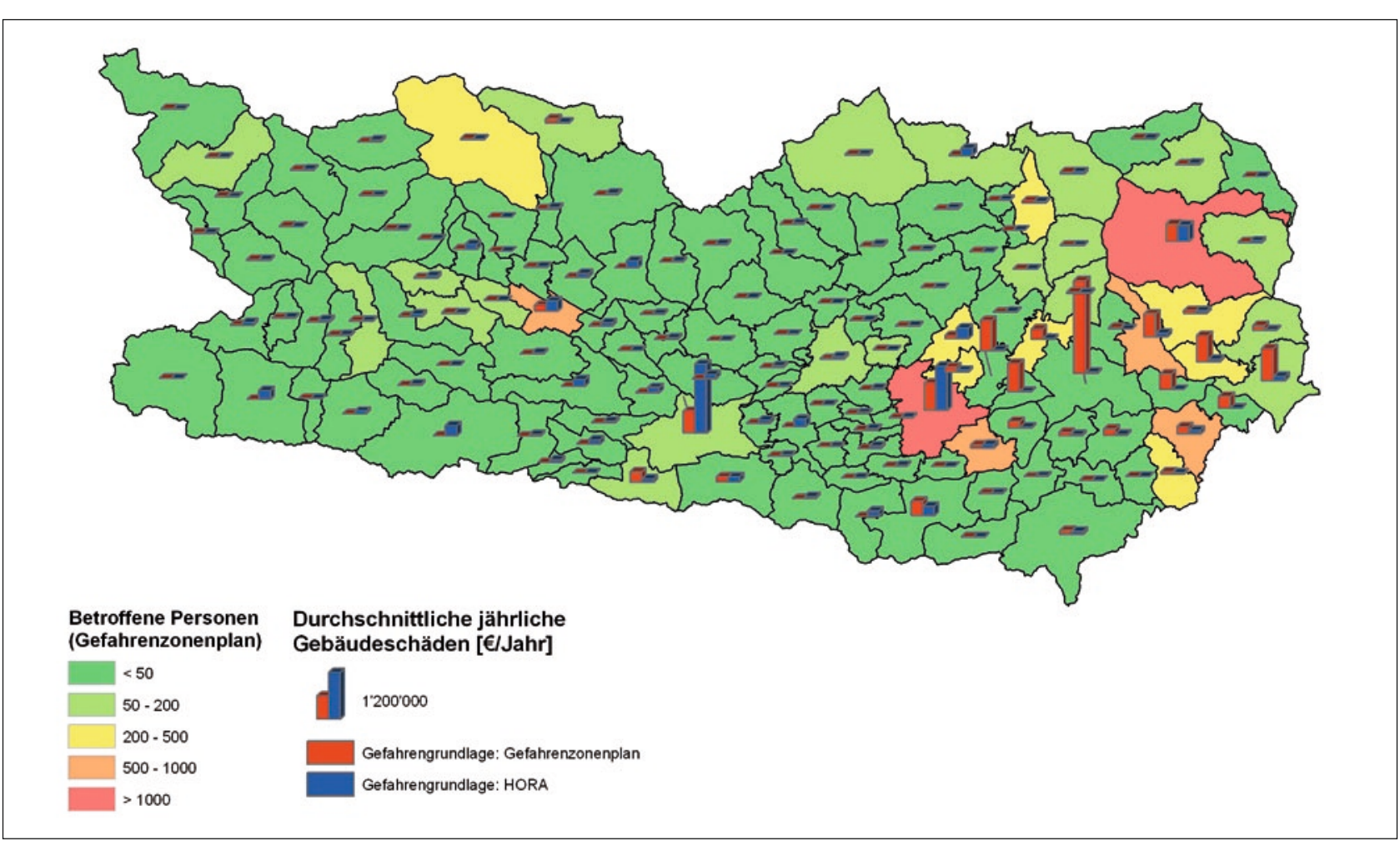

Abb. 5: Durchschnittliche jährliche Gebäudeschäden eines Hochwasserereignisses mit einer Wiederkehrperiode von 100 Jahren in Kärnten.

Schritt folgt die Klassifizierung der weiteren flächenhaften Objektkategorien wie Wald und landwirtschaftliche Flächen. Um die Anforderungen der EU-HWRL zu erfüllen, wird allen Datensätzen noch die Information hinzugefügt, ob sich das Objekt in einem Gebiet mit Schutzfunktion befindet.

Für die Erarbeitung des GIS-Datensatzes des Schutzgüterkataloges wurden sämtliche Geodatensätze verwendet, wobei versucht wurde, den Schutzgüterkatalog der EU-HWRL bestmöglich abzudecken (Tab. 1).

Nach Erstellung des GIS-Datensatzes des Schutzgüterkatalogs werden alle linienförmigen Objekte des Schutzgüterkatalogs aus den jeweiligen Grundlagendaten extrahiert, umklassifiziert und in einem einheitlichen Linien-Datensatz zusammengefügt. Für diesen Schritt werden das Straßennetz, das ländliche Wegenetz, das Bahnnetz, das Straßen- und Bahnnetz von Navteq, die Stromleitungen $(20 \mathrm{kV}$ und $110 \mathrm{kV}$ ) und die Gasleitungen verwendet.

Für die Erarbeitung des Punktdatensatzes des Schutzgüterkataloges werden die Wasserinfrastrukturanlagen sowie die Strom- und Gasanlagen verwendet.

Ergebnis dieser Prozedur sind ein Flächen-, ein Linien- und ein Punktdatensatz mit den nach der Tabelle des Schutzgüterkataloges klassifizierten räumlichen $\mathrm{Ob}$ - jekten. Diese Datensätze geben den Schutzgüterkatalog in einem ausgewählten Gebiet räumlich wieder.

Die zweite Prozedur überlagert den räumlichen Datensatz des Schutzgüterkataloges mit den existierenden Grundlagendaten zur Gefahrensituation im Sinne einer Expositions- oder Gefahrenanalyse. Je nach Fragestellung können die Gefahrenzonenpläne und Anschlaglinien der Bundeswasserbauverwaltung, die Gefahrenzonenpläne der Wildbach- und Lawinenverbauung und die Gefahrenhinweisbereiche der Landesgeologie einzeln oder gemeinsam von der Prozedur verarbeitet werden. Im letzteren Fall können auch die Objekte ermittelt werden, die mehreren Gefahrenarten ausgesetzt sind (Multi-Risiko-Situationen). Die Gebäude werden als Ganzes jener Intensitätsklasse eines Gefahrentyps zugeordnet, die die höhere Intensität aufweist. Ergebnis der Prozedur ist ein modifizierter räumlicher Datensatz des Schutzgüterkataloges, dessen Teilflächen oder Teilbereichen die Information der jeweiligen überlagernden Gefahrenzone als Attribut zugewiesen wird. Als zweites Ergebnis liefert die Prozedur der Gefahrenanalyse die Summe der von den verschiedenen Gefahrenzonen betroffenen Maßeinheiten je Kategorie des Schutzgüterkataloges. Für Gebäude sind dies die Anzahl Objekte und Flächenanteile des je- weiligen Nutzungstyps. Für sonstige flächenhafte Objektkategorien wird die Summe der je Gefahrenzonen betroffenen Flächen, für Linienobjekte werden die Laufmeter je Objektkategorie und Gefahrenzone berechnet. Für die Punktobjekte wird die Anzahl der Objekte je Gefahrenzone gezählt. Diese Ergebnisse werden in eine zusammenfassende Tabelle der Datenbank geschrieben.

Die dritte Prozedur der GIS-Applikation berechnet auf Basis der Ergebnisse der vorhergehenden Arbeitsschritte die potenziellen Schadenswerte und die Anzahl an betroffenen Personen und Beschäftigten im Gebäude. Für die Berechnung der Schadenswerte von Gebäuden wurden durchschnittliche B-Werte und minimale Schadenswerte je Gebäudetyp in Anlehnung an die KNU-Analyse der Bundeswasserbauverwaltung festgelegt. Vereinfachend wurde für die Rote Gefahrenzone eine durchschnittliche Wassertiefe von $0,77 \mathrm{~m}$ und für die Gelbe Zone eine Wassertiefe von $0,15 \mathrm{~m}$ angenommen. Die Schäden an den von HORA-Flächen betroffenen Gebäuden wurden mit einer durchschnittlichen Wassertiefe von $0,16 \mathrm{~m}$ berechnet.

Das Tool kann sowohl im lokalen Maßstab als auch im landesweiten Maßstab angewandt werden. Auf Gemeindeebene kann die Gefahrensituation nach den ver- 
schiedenen Prozesstypen unterschieden werden. Dieser „Gemeindesteckbrief“ gibt zunächst einen statistischen Überblick über die Gemeinde und fasst die durch Naturgefahren exponierten Werte zusammen, wobei die Gefahrensituation zusammenfassend in einer Karte dargestellt wird. Diese potenziellen Schäden werden sowohl in Tabellenform als auch in Form von Diagrammen dargestellt. In einem zweiten Schritt werden die Schäden und Risken nach Prozesstyp (Prozess Hochwasser, Wildbachprozesse und geologische Prozesse) unterschieden. Die Darstellung der Gefahrensituation auf Gemeindeebene und deren Auswirkungen soll der Gemeinde als Entscheidungsgrundlage bei raumplanerischen Tätigkeiten und Planungen innerhalb des Gemeindegebietes dienen ( $A b b .4)$. Die Monetarisierung erfolgte auf Basis von Mittelwerten aus der Kosten-Nutzen-Untersuchung der Bundeswasserbauverwaltung.

Für das Land Kärnten wurde mit den oben beschriebenen Vereinfachungen eine Analyse der Gebäudeschäden bei einem Hochwasserereignis mit einer Wiederkehrperiode von 100 Jahren durchgeführt ( $A b b$. 5). Da der Gefahrenzonenplan nicht für alle Gefahrengebiete vorhanden ist, wurden weitere - zum Gefahrenzonenplan komplementäre - Datensätze vorbereitet. Dort, wo kein Gefahrenzonenplan vorhanden war, wurden die Anschlaglinien oder die HORA-Flächen für die Gefahrenanalyse verwendet. Wo keine dieser drei Gefahrengrundlagen vorhanden war, wurde eine vereinfachte Abgrenzung der hochwassersensiblen Flächen durchge- führt (nach Sereinig 2007, Schober \& Zischg 2010). Diese Gefahrengrundlage repräsentiert ein extremes Ereignis ohne eine bestimmte Jährlichkeit. Abbildung 5 zeigt die Ergebnisse dieser vereinfachten Gefahrenanalyse auf der regionalen Maßstabsebene.

\section{Zusammenfassung}

Das Ziel des Risikomanagements ist es, potenzielle sich aus Naturgefahren ergebende negative Auswirkungen auf die Gesellschaft mittel- und langfristig zu minimieren. Hierzu müssen diese Risiken zunächst identifiziert, analysiert und bewertet werden, was es erforderlich macht, die Gefahrenanalyse um die Aspekte des Schadenpotenzials und der Verletzlichkeit $\mathrm{zu}$ erweitern. GIS-Systeme ermöglichen eine flächendeckende Erfassung dieser Risken. Das vorgestellte "GIS-Tool“ dient dabei als Hilfestellung und zeigt damit einen möglichen Weg, das Hochwasserrisiko im Sinne der EU-HWRL darzustellen. Das GIS-Tool erlaubt eine standardisierte
Erarbeitung eines räumlichen Datensatzes der schützenswerten Güter aus den bestehenden Datengrundlagen und eine Überlagerung mit den verfügbaren Gefahrenkarten. Das Tool kann einerseits die Grundlagen für eine detaillierte KostenNutzen-Untersuchung vorbereiten oder eine landesweite Übersicht über die potenziellen Schäden eines Gefahrenszenarios berechnen.

Die Ergebnisse bilden eine wichtige Entscheidungshilfe für eine objektive und transparente Prioritätensetzung der Maßnahmenplanung zum Schutz vor Naturgefahren und sind Grundlage für ein verbessertes Risikomanagement.

\section{Informationen:}

Amt der Kärntner Landesregierung

Abt. 18 - Wasserwirtschaft/Schutzwasserwirtschaft

DI Dr. Stephan Schober

Flatschacher Straße 70

9020 Klagenfurt

Tel. +43-463-536-31808

Fax +43-463-536-31828

stephan.schober@ktn.gv.at

http://wasser.ktn.gv.at

\section{LITERATUR}

Pleschko D, Schmid F, Sereinig N(2009) EUHochwasserrichtlinie und mögliche Auswirkungen auf die Gefahrenzonenplanung des Forsttechnischen Dienstes für Wildbach- und Lawinenverbauung, Zeitschrift

Keiler M, ZischgA, Fuchs S (2006) „Methoden zur GIS-basierten Erhebung des Schadenpotenzials für naturgefahreninduzierte Risiken", in: Strobl, J. und Roth, C.: GIS und Sicherheitsmanagement, Wichmann, Heidelberg, 118-128 Richtlinie 2007/60/EG des Europäischen Parlaments und des Rates über die Bewertung und das Management von Hochwasserrisiken
Schober S, Zischg A (2010) Naturgefahren Kärnten. Erstellung eines Gefahrenlayers in der Bundeswasserbauverwaltung - Methode und Umsetzung. Projektbericht im Rahmen des Projektes „AdaptAlp - Adaptation to Climate Change in the Alps" (Interreg Alpine Space) Change in

Sereinig N (2007) Der Schutzwasserwirtschaftliche Raumentwicklungsplan, SREP, Klagenfurt Seymann Ch, Sereinig N, Piechl T (2009) Naturgefahren Kärnten - Interdisziplinäre Methoden und neue Geodaten für ein effizientes Risikomanagement im alpinen Raum 